\title{
Traduire
}

Revue française de la traduction

$233 \mid 2015$

Voyage en équipage

\section{Pavese et les traducteurs d'Einaudi : un exemple de militantisme culturel sous le fascisme}

\section{Francesca Belviso}

\section{(2) OpenEdition}

Édition électronique

URL : http://journals.openedition.org/traduire/767

DOI : $10.4000 /$ traduire. 767

ISSN : 2272-9992

Éditeur

Société française des traducteurs

Édition imprimée

Date de publication : 15 décembre 2015

Pagination : 85-95

ISSN : 0395-773X

\section{Référence électronique}

Francesca Belviso, «Pavese et les traducteurs d'Einaudi : un exemple de militantisme culturel sous le fascisme », Traduire [En ligne], 233 | 2015, mis en ligne le 15 décembre 2017, consulté le 24 avril 2019 URL : http://journals.openedition.org/traduire/767 ; DOI : 10.4000/traduire.767 


\section{Pavese et les traducteurs d'Einaudi : un exemple de militantisme culturel sous le fascisme}

\section{Francesca Belviso}

L'œurre de transmission et d'opposition culturelle réalisée sous le fascisme par Cesare Pavese, romancier, poète, essayiste et traducteur parmi les plus reconnus du xxe siècle, constitue un chapitre important dans l'histoire de la culture italienne moderne(1). Cette stratégie d'opposition s'insère dans un discours plus vaste portant sur le " militantisme culturel turinois ". Cette formule choisie par Eugenio Garin(2) fait référence aux stratégies adoptées par un certain milieu culturel de Turin et, plus précisément, par un groupe d'auteurs-traducteurs antifascistes dont Pavese fut l'un des membres les plus prolifiques. Dans la capitale piémontaise de l'entre-deuxguerres, ces jeunes intellectuels vont former l'une des sociétés les plus brillantes de la scène culturelle italienne. Turin offre, de ce point de vue, un panorama d'une richesse inépuisable : creuset de nouveautés et lieu privilégié de la quête de la modernité, la ville accueille et nourrit à cette époque les esprits vifs et engagés d'une prometteuse génération de militants. Des personnalités aussi singulières que diverses se sont formées et mutuellement influencées dans ces années troubles : des écrivains-traducteurs tels que Cesare Pavese, Carlo Levi, Mario Soldati, Natalia Ginzburg ou Lalla Romano ; le musicologue Massimo Mila ; des historiens et critiques littéraires tels que Natalino Sapegno, Giacomo Debenedetti, Carlo Dionisotti ou Norberto Bobbio. À la tête de cette insigne compagnie de chevaliers de la future intelligentsia italienne trône le spécialiste de la langue et de la littérature russes, mort prématurément dans les geôles fascistes, Leone Ginzburg(3). La vivacité de ces jeunes intellectuels, éduqués aux valeurs du libéralisme et de l'idéalisme de Croce, imprégnés du sens civique laissé en héritage par Gramsci et recueillant la leçon morale de Gobetti, vont constituer une nouvelle génération appelée gobettiana(4). Après la disparition de Piero Gobetti, mort à Paris en 1926, cette génération se réunit autour de la revue // Baretti dans le même esprit que celui qui avait animé

(1) Cf. PAvese Cesare, 1999, p. 225-227.

(2) Garin Eugenio, 1962.

(3) Voir d'ORSI Angelo, 1996, p. 68-111.

(4) Du nom de Piero Gobetti, fondateur, en 1924, de la revue // Baretti, l'une des premières revues turinoises engagées sous le fascisme. 
son fondateur : le renouvellement moral, l'attention à toute nouvelle tendance artistique ; le refus d'une littérature strictement nationale et l'ouverture à la littérature étrangère. En d'autres termes, dans la lignée idéologique lancée par Gobetti, // Baretti inaugure une tradition journalistique de militantisme culturel profondément ancrée dans la réalité urbaine de l'ancienne capitale du royaume d'Italie. Sur l'existence d'une culture militante ayant réussi à survivre à Turin grâce aux stratégies adoptées afin d'échapper à l'emprise du régime, il existe désormais une vaste et solide littérature critique. L'une de ces stratégies, reconnue d'ailleurs par tous les historiens, s'identifie dans la vocation à ce que Garin définit comme l'europeismo de la génération gobettienne. Les nombreux articles sur la littérature étrangère parus dans les pages de plusieurs revues turinoises, ainsi que la traduction et la diffusion d'ouvrages réalisées par une véritable floraison de maisons d'éditions (Slavia, Frassinelli, Einaudi, pour ne citer que les plus connues) représentent les étapes d'une "déprovincialisation " progressive de la culture italienne dominante. Comme le souligne Bobbio dans un ouvrage fondateur sur la culture à Turin dans les années 1930, la génération turinoise de l'entre-deux-guerres est partagée entre le sentiment d'appartenir à une tradition littéraire nationale, désormais ressentie comme " asphyxique ", et la recherche d'un idéal d'ouverture à la modernité incarnée par la littérature étrangère, surtout française, anglaise et russe(5). L'une des stratégies d'opposition les plus efficaces de cette génération d'antifascistes, l'européisme, s'est donc soldée par l'intense travail de traduction et de transmission d'ouvrages de littérature étrangère réalisé sous le régime mussolinien et après sa chute(6).

Jeune diplômé de l'université de Lettres et Sciences humaines de Turin en 1930 avec un mémoire de maîtrise sur la poésie de Walt Whitman, Pavese semble avoir d'abord hésité sur le choix d'une carrière universitaire. II sollicita en vain un poste d'assistant de littérature italienne à la Faculté des Lettres de Turin(7) puis, de 1930 à 1932, tenta, sans succès, d'obtenir une bourse à la Columbia University de New York(8). Son diplôme d'études supérieures en poche, le jeune écrivain commença à traduire, d'abord à son compte, puis pour différentes revues et maisons d'édition, de nombreux ouvrages anglo-américains totalement inconnus du public italien : en 1931 Our Mr. Wrenn de Sinclair Lewis, en 1932 Moby Dick d'Herman Melville et Dark Laughter de Sherwood Anderson, en 1933 Dedalus de James Joyce, en 1934 The 42nd Parallel de John Dos Passos. II fit paraître en même temps plusieurs études critiques sur la plupart de ces auteurs : sur Lewis (1930 et 1934), Anderson (1931 et 1932), Lee Masters (1931), Melville (1932), 0. Henry (1932), Dos Passos (1933), Whitman (1933), Dreiser (1933), Faulkner (1934). Comme il ne maîtrisait pas encore parfaitement l'anglo-américain, il fit appel à l'aide d'un Piémontais émigré aux États-Unis depuis 1908, Antonio Chiuminatto. La

(5) BoBBio Norberto, 1977, p. 66.

(6) Voir, à ce propos, d'Orsi Angelo, 2000, p. 73-96.

(7) Cf. la lettre à son professeur de lettres Augusto Monti du 11 octobre 1930. PAVESE Cesare, 1966, Lettere 19241944, p. 244.

(8) Cf. la lettre envoyée à l'université américaine le 13 juin 1930. Ibid., p. 204. 
correspondance que le jeune auteur-traducteur tissa avec cet émigré à Chicago (une trentaine de lettres au total, entre 1929 et 1933) témoigne d'un riche travail d'apprentissage linguistique, mais atteste également une passion littéraire nourrie depuis le plus jeune âge. Les lettres à Chiuminatto offrent en outre de précieuses indications sur les goûts et les intérêts littéraires du traducteur autodidacte qui déclarait préférer, par-dessus tout, les écrivains américains comme Melville, Whitman, Lee Masters et Anderson, pour des raisons précises qu'il aura l'occasion d'exposer dans plusieurs essais publiés entre 1932 et 1947.

Dans l'Italie de l'entre-deux-guerres, l'apprentissage de langues et de littératures étrangères, plus particulièrement anglo-saxonne et américaine, ne rentrait pas systématiquement dans les programmes d'enseignement du secondaire et du supérieur, à l'exception du français qui était toujours, depuis l'époque napoléonienne, la seconde langue pour les milieux intellectuels et les classes les plus aisées. Le choix de Pavese, dont l'apprentissage de la langue anglaise s'inscrivait dans un parcours singulier, voire expérimental, était donc loin d'être anodin : sa valeur était éminemment politique. Ce fut seulement à partir de la moitié des années trente(9) que la passion de Pavese se transforma en véritable profession au sein d'Einaudi, l'une des maisons d'édition italiennes les plus influentes pendant et après la dictature mussolinienne. Cette maison d'édition reprenait le flambeau laissé par deux grandes entreprises journalistiques interdites par le régime : Solaria et La Cultura. La première de ces revues avait été arrêtée en 1934 par un décret préfectoral et la seconde en 1935, toutes deux accusées de véhiculer des propos contraires à la morale publique et à la culture fasciste. L'arrêt de Solaria a coïncidé avec le moment où, à partir des années 1934-1935, le tournant idéologique engagé par son fondateur Carocci se révélait pleinement à travers sa transformation en une véritable "revue d'idées et de confrontation avec le régime "(10). Cette revue dressait l'étendard de l'antifascisme en ouvrant ses colonnes aux courants les plus modernes de la littérature européenne. Comme le rappelle le grand romancier Elio Vittorini, être solarien signifiait, à l'époque, être un farouche antifasciste et un européen facilement traité de "sale juif ", de "chacal " ou de "fossoyeur ", lorsque la revue publiait et soutenait des écrivains de religion hébraïque tels que Kafka ou Joyce(11).

Après la fermeture officielle de Solaria, La Cultura s'engagea dans la lutte en polarisant autour d'elle tout le milieu antifasciste turinois de l'entre-deux-guerres(12). La principale caractéristique de cette revue jusqu'en 1935 était, en plus d'un antifascisme diffus, une certaine opposition à la philosophie idéaliste de Croce qui s'exprimait notamment par une tentative de restauration du comparatisme condamné par la critique crocienne.

(9) La maison d'édition naît officiellement en 1933, mais le contrat est signé par Pavese avec l'éditeur seulement quelques années plus tard.

(10) TuRI Gabriele, 1986, p. 79-100.

(11) Cf. VITTORINI Elio, 1999.

(12) Giulio Einaudi a été assigné à résidence surveillée de 1935 à 1942. 
La collaboration de Pavese avec cette revue débuta officiellement en 1934 avec un rôle de premier plan : sa direction. II s'agissait en réalité d'un simple rôle de prête-nom qu'on lui avait confié puisqu'il était l'un des rares collaborateurs à être officiellement inscrit au Parti fasciste depuis 1932. Cette fonction, qu'il aurait acceptée " bon gré, mal gré "(13), dura en tout cas moins d'un an car il fut arrêté en mai 1935, son activité culturelle étant jugée subversive et hostile au régime(14). En tout état de cause, les jeunes Turinois antifascistes rassemblés essentiellement autour de Ginzburg, Pavese et Einaudi décidèrent de se lancer dans une activité éditoriale de vaste envergure. Ils allaient fonder une véritable maison d'édition dont l'emblème était à lui seul un manifeste : une autruche avalant un clou. L'image s'accompagnait de l'inscription latine Spiritus durissima coquit, indiquant clairement que seule la force de l'esprit permet, par les temps les plus sombres, de tout endurer. À partir de 1938, date officielle de la signature de son contrat chez Einaudi, Pavese allait acquérir une position de premier plan au sein de la maison d'édition, mais au prix d'un travail énorme, voire harassant(15). II devait réaliser des traductions de l'anglais et de l'anglo-américain (environ deux mille pages par an), examiner les manuscrits et les épreuves d'ouvrages étrangers et italiens, fonder de nouvelles collections, rédiger des comptes rendus, s'occuper de la correspondance courante ; tout cela parallèlement à son activité de romancier et de poète. De 1938 à 1947 il traduisit pour Einaudi une douzaine d'ouvrages de l'anglais, mais le chiffre serait bien plus élevé si l'on tenait compte du nombre très important de ses traductions inachevées de l'anglais, du grec, du latin, mais également de l'allemand(16).

Au croisement entre " religion de la liberté " d'inspiration crocienne et de tradition gobettienne, se plaçait également l'activité de l'autre pilier de la maison d'édition, Leone Ginzburg, qui eut un rôle déterminant dans sa fondation(17). Ce spécialiste de la langue et de la littérature russes a par ailleurs exercé la plus grande influence sur les membres du groupe gobettien en général et sur Pavese en particulier. Né en 1909 à Odessa, mais installé depuis l'enfance en Italie, il

(13) Voir, à ce propos, le témoignage de l'éditeur sur Pavese, in EInAudi Giulio, 1988, p. 43-45. Voir également la lettre de Pavese à Giulio Einaudi du 13 janvier 1935, in PAVESE Cesare, 1966, Lettere 1924-1944, p. 376.

(14) Pavese, arrêté en mai 1935, passe d'abord un mois dans la maison d'arrêt de Turin puis est envoyé à Rome dans la prison de Regina Coeli où il y demeure jusqu'à fin juillet. Condamné à trois années d'assignation à résidence surveillée à Brancaleone Calabro, en Calabre (sud de l'Italie), il est relaxé en mars 1936 à la suite d'une demande de grâce adressée à Mussolini.

(15) Voir, à ce propos, la lettre de Pavese à son ami Tullio Pinelli du 4 décembre 1939 dans laquelle il se plaint d'être exploité comme " un esclave égyptien ». PAVESE Cesare, 1966, Lettere 1924-1944, p. 549.

(16) Pavese a été un fin lecteur et traducteur de l'allemand, notamment de Nietzsche. Cf. Francesca Belviso, Pavese face à Nietzsche. Physiologie d'une "métaphysique d'artiste ", Thèse de doctorat en Études italiennes dirigée par Denis Ferraris et Michel Paoli, Paris 3 Sorbonne Nouvelle (592 p.). Tous les manuscrits pavésiens sont conservés au Centro interuniversitario per gli studi in Piemonte "Guido Gozzano-Cesare Pavese " de l'Université de Turin.

(17) C'est à lui que reviendrait la véritable paternité de la maison d'édition Einaudi. Voir, à ce propos, d'ORSı Angelo, 2001 , p. 344. 
eut un ascendant prépondérant sur plusieurs membres de la bande qui n'ont jamais hésité à souligner ses qualités humaines exceptionnelles et sa grande vivacité intellectuelle. Du haut de sa précoce maturité, celui que l'on nommait habituellement "Agence Tass" (en raison de son origine russe, mais surtout parce qu'il semblait tout savoir sur les sujets les plus divers) a eu une place capitale non seulement dans l'histoire des groupes intellectuels antifascistes, mais, plus largement, dans l'histoire culturelle italienne de l'entre-deux-guerres. Dans les pages de La Cultura, Ginzburg révéla aux lecteurs italiens de nombreux écrivains russes en publiant, en traduction italienne, des ouvrages tels que Tarass Boulba de Gogol, Anna Karénine de Tolstoï, La Dame de pique de Pouchkine et Une nichée de gentilshommes de Tourgueniev. De 1927 à 1934 il écrivit dans différentes revues (/I Baretti et La Cultura entre autres) une série d'articles sur Gogol, Gontcharov, Tourgueniev, Dostoïevski, Tolstoï, Leskov, Garchine, Tchékhov et Gorki. En 1930, fort de la reconnaissance et de l'influence dont il jouissait déjà auprès des professeurs de l'université, il intervint auprès du corps enseignant pour que Pavese eût le droit de présenter son mémoire de maîtrise sur Walt Whitman, jugé trop original. L'intense activité de traduction menée en synergie par les deux hommes au cours de ces années troubles constitue un modèle saisissant d'engagement intellectuel audacieux qui s'interrompit tragiquement en mars 1944, lorsque Pavese, caché sous une fausse identité sur les collines piémontaises, apprit la disparition de son ami. La mort de Ginzburg, survenue fort probablement à la suite des maltraitances subies en prison, a été sans aucun doute une perte irréparable pour Pavese qui voyait en lui, de son propre aveu, l'un de ses amis les plus chers, voire son seul véritable compagnon de route(18).

L'engagement ouvertement antifasciste n'était pas une marque de fabrique clairement définie pour tous les collaborateurs de la maison d'édition Einaudi. Ses deux piliers, Ginzburg et Pavese, entretenaient en effet des rapports bien distincts à la politique. Si Ginzburg fut un "vrai militant "(19) (il a d'ailleurs dirigé le groupe antifasciste Giustizia e Libertà), l'engagement politique de Pavese relèverait plutôt d'un long travail éditorial(20). La problématique de l'engagement politique de Pavese a été abordée par une abondante littérature critique qui a parfois grossi les traits d'un homme de lettres enfermé dans sa tour d'ivoire. Des interprétations hâtives, ne tenant pas compte du contexte historique, ont contribué à transmettre une image parfois faussée de l'auteur(21). Pour Calvino, bien que Pavese ait été essentiellement " inapte à la vie politique "(22), son discours littéraire serait, depuis le début, " un discours

(18) Voir, à ce propos, la note écrite dans son journal intime à la date du 3 mars 1944. PAVESE Cesare, 2008, p. 1654. PAVESE Cesare, 1990, p. 276.

(19) TuRI Gabriele, 1990, p. 20.

(20) Voir d'ORSI Angelo, 2010, p. 129.

(21) Dans cette perspective, l'ouvrage qui a eu une influence durable dans l'histoire de la critique pavésienne est celui de GuIDucCl Armanda, 1967.

(22) Cf. la lettre d'Italo Calvino à Valentino Gerratana du 15 septembre 1950, in SPRIAno Paolo, 1986, p. 38. 
également politique "(23) dans la mesure où l'ouverture à la littérature étrangère, en particulier américaine, témoignait d'une opération allant à l'encontre des politiques culturelles dominantes sous le fascisme. En effet, si les premières traductions pavésiennes étaient le fruit d'un travail et d'une passion tout à fait personnelles, l'activité du traducteur devint, au fur et à mesure que le régime fasciste se mettait en place, une manière de chercher une nouvelle forme d'expression au sein d'une autarcie culturelle de plus en plus étouffante. Les nombreuses traductions d'ouvrages appartenant à la sphère américaine ou anglo-saxonne devenaient donc de plus en plus, pour le jeune homme de lettres, un acte de subversion de la culture italienne dominante, comme en témoigne sa camarade Fernanda Pivano, elle-même traductrice de plusieurs ouvrages de l'anglo-américain(24).

S'il est donc indéniable que l'ouverture à la littérature étrangère ait été pour Pavese une manière de créer un espace de liberté au sein de l'étouffante autarcie du régime, cela a été bien plus encore l'occasion pour le jeune écrivain de trouver enfin sa voie, au moins au tout début de son parcours de formation. Dans cette perspective, il n'est pas inutile de rappeler un témoignage saisissant qui date de 1945 :

[...] Mais nous n'avons réussi à lire que peu de livres italiens pendant les journées tapageuses de l'ère fasciste, pendant cette absurde vie oisive, repliée sur elle-même, qu'il nous a fallu mener alors, et nous avons rencontré des hommes plus que des livres, nous avons rencontré le sang et la chair d'où naissent les livres. Dans nos efforts pour comprendre et pour vivre, des voix étrangères nous ont soutenus : chacun de nous a fréquenté et aimé d'amour la littérature d'un peuple, d'une société lointaine, il en a parlé, il en a traduit des textes, il s'en est fait une patrie idéale. Dans le langage fasciste, tout cela s'appelait xénophilie. Les modérés nous accusaient de vanité exhibitionniste, et d'exotisme prétentieux, les rigoristes disaient que nous cherchions dans les goûts et les modèles qui nous venaient d'au-delà des océans ou des Alpes un défoulement pour notre indiscipline sexuelle ou sociale. Naturellement, ils ne pouvaient admettre que nous cherchions en Amérique, en Russie, en Chine ou Dieu sait où, une chaleur humaine que l'Italie officielle ne nous donnait pas. Moins encore que nous, nous cherchions tout simplement nous-mêmes.(25)

L'opposition politique de Pavese s'avère, certes, essentiellement culturelle(26) dans la mesure où, comme le confirme l'écrivain dans un article publié en 1947, la rencontre intellectuelle avec des génies tels que Caldwell, Steinbeck ou Faulkner ouvrait des perspectives de liberté et donnait l'espoir que la vraie culture ne s'arrêtait pas à la littérature nationale(27). Pour

(23) Calvino Italo, Prefazione in PAvese Cesare, La letteratura americana e altri saggi, p. 23.

(24) Voir Pivano Fernanda, 1995.

(25) PAVESE Cesare, "Retour à l'homme ", in Id., Littérature et société, p. 33-34. PAVESE Cesare, "Ritorno all'uomo ", in Id., La letteratura americana e altri saggi, p. 217.

(26) FERME Valerio Cristiano, 1998.

(27) PAVESE Cesare, "L'influence des événements ", in Id., Littérature et société, p. 69-74. PAVESE Cesare, "L'influsso degli eventi ", in Id., La letteratura americana e altri saggi, p. 245-248. 
Pavese, le recours à la littérature américaine apparaissait donc nécessaire, voire vital face au sentiment d'ingérence du régime fasciste. II s'agissait toutefois d'une opposition purement esthétique, bornée au domaine de la littérature et qui ne s'adressait qu'à cette partie de lecteurs italiens ouverts au renouvellement d'une culture italienne sclérosée dans l'enfermement nationaliste. Le peuple américain, garant d'une nouvelle forme de liberté et d'une soif de démocratie, devenait ainsi l'emblème de la quête de l'indépendance, tout en étant le miroir de la situation politique italienne. La culture américaine reflétait, somme toute, une réalité proche et constituait ainsi le modèle précieux d'un laboratoire où les individus poursuivaient tous le même objectif : celui de comprendre un réel devenu de plus en plus opaque; de créer un goût et un style véritablement nouveaux. C'est dans cette perspective que la culture américaine constitua, pour Pavese comme pour d'autres auteurs-traducteurs de sa génération tels que Vittorini, un héritage précieux qu'il fallait sauver et transmettre à tout prix, puisque l'Amérique, plus que tout autre continent, représentait un immense "théâtre " où la liberté d'expression de chacun pouvait s'exercer sous les formes les plus riches, diverses et modernes(28).

Le lien entre la culture et la politique se solda chez Pavese par une stratégie fondamentale qui consistait à vacciner les lecteurs italiens contre tout régionalisme pouvant facilement dégénérer en xénophobie aveugle ; l'ouverture aux différents horizons de la modernité culturelle européenne permettait ainsi de chasser " la puanteur du nationalisme et du racisme "(29). II s'agissait donc d'une opération de subversion culturelle qui ne cherchait pas la confrontation directe et brutale, mais qui s'efforçait de changer les mentalités de l'intérieur en mettant en lumière une nouvelle constellation littéraire capable de redonner le goût de la liberté et de la diversité. La maison d'édition Einaudi a donc été pour l'écrivain turinois un véritable laboratoire intellectuel dans lequel se reflétaient ses lectures et ses intérêts les plus variés dans l'objectif de garder toujours un lien très fort avec la société de son temps, afin de ne pas rester en dehors des événements(30). Toutefois, selon Asor Rosa, Pavese avait peu de chances de porter ses idées en dehors de cette étroite "fraction " de la maison d'édition qu'il semblait contrôler directement(31). En réalité, Pavese portait en lui l'héritage intellectuel laissé par Gobetti et Monti, ses premiers maîtres à penser(32). Pour eux, l'une des manières de lutter activement contre l'autarcie du régime fasciste était de faire barrage à la culture dominante en proposant une palette riche et variée de références culturelles. S'inscrivant pleinement dans ce sillage, Pavese considérait que s'éduquer à travers la lecture traduisait, tout d'abord, le refus de toute forme d'oisiveté

(28) Ibid., p. 73.

(29) DionisotTi Carlo, p. 16.

(30) PAVESE Cesare, "Le communisme et les intellectuels ", in Id., Littérature et société, p. 47-62. PAVESE Cesare, " II comunismo e gli intellettuali ", in Id., La letteratura americana e altri saggi, p. 229-239.

(31) Asor Rosa Alberto, 1982, p. 585.

(32) DugheRA Attilio, 1992, p. 49-103. 
intellectuelle. Vivre en intellectuel engagé signifiait, dans l'enseignement de Monti(33), essayer de comprendre le réel en questionnant sans cesse l'Histoire, sans se contenter de réponses toutes faites. Enfin, l'homme de lettres avait pour véritable mission de s'ouvrir à un maximum d'expériences intellectuelles et humaines, afin de susciter chez les lecteurs le goût de la découverte. Cette découverte pouvait passer par celle de nouvelles formes de langage. Ainsi, le choix d'un langage particulier conférait-il, à lui-seul, le pouvoir de poser une interrogation d'ordre politique :

Certes tout est langage chez un écrivain qui mérite ce nom, et justement il suffit de l'avoir compris pour se trouver dans un monde très vivant et complexe où le choix d'un mot, d'une inflexion, d'un rythme, devient aussitôt un problème de mœurs, de moralité. Ou carrément de politique. Arrêtons là. Ce que l'on appelle l'art est une chose sérieuse. Au moins aussi sérieuse que la morale ou la politique(34).

S'il est donc vrai que la décennie 1930-1940 fut celle des traductions de l'anglo-américain dans I'histoire culturelle italienne, ce ne fut ni le fruit du hasard, ni celui d'un désœuvrement des classes intellectuelles. Bien au contraire, puisque l'Italie était devenue étrangère à elle-même : " il fallait la secouer, la décongestionner, l'exposer à nouveau à tous les vents printaniers de l'Europe et du monde „(35). La littérature américaine constituait, en fin de compte, le principal centre d'intérêt de Pavese à cause des valeurs éminemment modernes du peuple américain, peuple neuf par excellence, capable de réinventer une langue, un style, une vision du monde avec toute l'énergie d'un peuple de "producteurs "(36).

Après son intense activité de traducteur et de conseiller, Pavese assuma, notamment à partir de 1945, un véritable rôle de dirigeant au siège turinois. La marque de reconnaissance exprimée par son ami Vittorini à l'occasion de sa mort apparaît particulièrement significative à cet égard : "La maison d'édition Einaudi se fondait sur son travail "(37). Son engagement, s'étalant sur quinze années de travail infatigable, a donc été l'expression directe de cette " morale de l'action " dont a si bien parlé Calvino dans sa tentative de soustraire Pavese à l'image plate d'intellectuel suicidaire, incapable de résister à son insupportable élan autodestructeur(38). Pendant et après la guerre, l'écrivain fit le choix de participer activement au travail collectif de la maison d'édition Einaudi dans l'intention de coopérer à la construction d'un nouvel

(33) Pour un témoignage saisissant sur l'héritage intellectuel de Monti, voir MILA Massimo, 1949, V, p. 1136-1148, et MILA Massimo, 1992, p. 303-321. Pour une biographie d'Augusto Monti, voir TesIo Giovanni, 1980.

(34) PAVESE Cesare, "Lire ", in Id., Littérature et société, p. 41. PAVESE Cesare, "Leggere ", in Id., La letteratura americana e altri saggi, p. 223-224.

(35) PAvESE Cesare, "L'influence des événements ", in Id., Littérature et société, p. 73. PAvESE Cesare, "L'influsso degli eventi ", in Id., La letteratura americana e altri saggi, p. 247.

(36) L'expression " civilisation des producteurs " est employée pour la première fois par un autre membre du groupe d'Einaudi. Voir PINTOR Giaime, 1965.

(37) Voir la lettre d'Elio Vittorini à son père du 12 septembre 1950, in VITTORINI Elio, 1977, p. 330.

(38) Calvino Italo, 1980, "Pavese: essere e fare ", in Id., Una pietra sopra. Discorsi di letteratura e società, p. 60. 
ordre économique et social, afin d'atteindre " un bien-être collectif qui est le fruit d'un travail collectif „(39). En jouant avec passion et conscience le rôle de médiateur que Giulio Einaudi lui conféra au sein de sa maison, Pavese réalisa sans nul doute l'une des entreprises les plus importantes dans l'histoire de la culture italienne : il sut théoriser et incarner l'" éclectisme " politique et culturel de l'Italie fasciste et postfasciste dans la perspective d'un militantisme culturel et d'un esprit collégial qui étaient la marque de reconnaissance de l'aventure éditoriale de "l'Autruche " depuis sa fondation en 1933 jusqu'à la mort de l'écrivain-traducteur en 1950(40).

francebelviso@hotmail.com

\section{Francesca Belviso}

Professeur de langue et culture italiennes à l'UFR de Langues et Cultures étrangères de l'Université de Picardie Jules Verne. Historienne de la culture italienne de la première moitié du xxe siècle, spécialiste des groupes intellectuels et des mouvements du militantisme culturel turinois. Auteure d'une thèse sur Cesare Pavese (Pavese face à Nietzsche. Physiologie d'une "métaphysique d'artiste ") et traductrice, entre autres, de J.M.G. Le Clézio en Italie. Son dernier ouvrage Amor Fati. Pavese all'ombra di Nietzsche (204 p.) vient de paraître chez Nino Aragno Editor.

(39) Voir la lettre de Cesare Pavese à Silvio Micheli du 11 juin 1945 in PAVESE Cesare, 1966, Lettere 1945-1950, p. 15.

(40) Pavese s'est suicidé dans la nuit du 27 août 1950 dans une chambre d'hôtel, à Turin, à l'âge de quarantedeux ans. Pour un éclairage exhaustif sur l'importance de son activité éditoriale, je renvoie à l'ouvrage monumental de MANGONI Luisa, 1999. 


\section{Bibliographie}

Asor Rosa Alberto, 1982, Lo Stato democratico e i partiti politici, in Letteratura italiana, vol. I, Il letterato e le istituzioni, Torino (Italie), Einaudi.

Boвві0 Norberto, 1977, Trent'anni di storia della cultura a Torino (1920-1950), Torino (Italie), Einaudi, Cassa di Risparmio di Torino.

Calvino Italo, 1980, Una pietra sopra. Discorsi di letteratura e società, Torino (Italie), Einaudi. CALvino Italo, 1962, Prefazione in PAvESE Cesare, La letteratura americana e altri saggi, Torino (Italie), Einaudi, p. 11-33.

DIonISOTtI Carlo, s.d., "Culture regionali e letteratura nazionale in Italia ", in Atti del VII Congresso di Bari, 31 marzo-4 aprile 1970, Associazione internazionale per gli Studi di Lingua e Letteratura italiana, Bari (Italie), Adriatica Editrice, p. 13-27.

D'ORSI Angelo, 2010, "Pavese e Ginzburg. Due modelli di intellettuale ", in Brandone Giorgio, Cerrato Tiziana (éd.), Incontro con Cesare Pavese. Un giorno di simpatia totale, Atti del convegno di studi Convitto Nazionale Umberto I - Liceo Classico D’Azeglio, Torino, 23-24 ottobre 2008, Torino (Italie), Quaderno n. 3, Liceo Classico «D'Azeglio », p. 123-142.

D'ORSI Angelo, 2001, Intellettuali nel Novecento italiano, Torino (Italie), Einaudi.

D'ORSI Angelo, 2000, La cultura a Torino tra le due guerre, Torino (Italie), Einaudi.

D'OrSI Angelo, 1996, "Un suscitatore di cultura ", in TranfagliA Nicola (éd.), L'itinerario di Leone Ginzburg, Torino (Italie), Bollati Boringhieri, p. 68-111.

Dughera Attilio, 1992, Tra le carte di Pavese, Roma, Bulzoni.

EInAudi Giulio, 1988, Frammenti di memoria, Milano (Italie), Rizzoli.

Ferme Valerio Cristiano, 1998, Cesare Pavese's and Elio Vittorini's translations from American literature: the americanization of aesthetic and the subversion of culture under the Fascist regime (Italy), Berkeley (États-Unis), University of California.

GARIN Eugenio, 1962, La cultura italiana tra '800 e '900. Studi e ricerche, Bari (Italie), Laterza. Guiduccl Armanda, 1967, II mito Pavese, Firenze (Italie), Vallecchi.

MAngoni Luisa, 1999, Pensare i libri. La casa editrice Einaudi dagli anni trenta agli anni sessanta, Torino (Italie), Bollati Boringhieri.

MııA Massimo, 1992, Scritti civili, a cura di Alberto Cavaglion, Torino (Italie), Einaudi.

MILA Massimo, 1949, "Augusto Monti educatore e scrittore ", in II Ponte, V, p. 1136-1148. 
PAvese Cesare, 2008, Le Métier de vivre 1935-1950, édition intégrale établie sur manuscrit accompagnée d'un choix de lettres, traductions de Michel Arnaud et Gilbert Moget (nouvelles traductions et révisions par Martin Rueff), in Id., OEuvres, Paris, Gallimard, "Quarto ".

PAVESE Cesare, 1999, Littérature et société suivi de Le mythe, traduit de l'italien et préfacé par Gilles de Van, Paris, Gallimard, "Arcades ".

PAVESE Cesare, 1990, Il mestiere di vivere. Diario 1935-1950, edizione condotta sull'autografo a cura di Marziano Guglielminetti e Laura Nay, Torino (Italie), Einaudi.

Pavese Cesare, 1966, Lettere 1924-1944, a cura di Lorenzo Mondo, Torino (Italie), Einaudi.

PAVESE Cesare, 1966, Lettere 1945-1950, a cura di Italo Calvino, Torino (Italie), Einaudi.

PAVESE Cesare, 1962, La letteratura americana e altri saggi, Torino (Italie), Einaudi.

PInTOR Giaime, 1965, Il sangue d'Europa (1939-1943), a cura di Valentino Gerratana, Torino (Italie), Einaudi, (1re éd. 1950).

Pivano Fernanda, 1995, La Balena bianca ed altri miti, Milano (Italie), II Saggiatore.

SPRIAno Paolo, 1986, Le passioni di un decennio :1946-1956, Milano (Italie), Garzanti.

Tesıo Giovanni, 1980, Augusto Monti. Attualità di un uomo all'antica, Cuneo (Italie), L'Arciere.

TuRI Gabriele, 1990, Casa Einaudi. Libri, uomini, idee oltre il fascismo, Bologna (Italie), II Mulino.

TURI Gabriele, 1986, "Lo spazio di Solaria ", in Manghettı Gloria (éd.), Gli anni di Solaria, Verona (Italie), Bi \& Gi, p. 79-100.

VITTORINI Elio, 1999, Diario in pubblico, Milano (Italie), Bompiani.

VItTorinı Elio, 1977, Gli anni del «Politecnico ». Lettere 1945-1951, Torino (Italie), Einaudi. 